O25 (continued)

barriers and opportunities for PSE capacity building within the state's SNAP-Ed program, and preferred PSE strategies among stakeholders.

Results: IA staff's knowledge of PSE requirements increased during the year. Numerous barriers (e.g., lack of resources and familiarity of strategies) and opportunities (e.g., current relationships and interest in strategies) for capacity building were identified. Some preferred PSE strategies aligned among stakeholder groups (e.g., farmers markets, gardens).

Conclusions and Implications: Opportunities exist to build capacity of SNAP-Ed programs to comply with federal rules through exploring and aligning the readiness and need of IAs, partners, and target populations.

Funding: Supplemental Nutrition Assistance Program Education

\section{Mandela MarketPlace's Health and Wealth Net: A Resident-Led, Systems-Based Food Enterprise Model}

TrishaChakrabarti, MPH, MS, trisha@

mandelamarketplace.org, Mandela MarketPlace, 1364 7th

Street, Oakland, CA 94607; Janice Kao, MPH, Nutrition

Policy Institute, University of California Division of

Agriculture and Natural Resources; Dana Harvey, MS, Mandela MarketPlace; Gail Woodward-Lopez, MPH, RD, Nutrition Policy Institute, University of California Division of Agriculture and Natural Resources

Objective: Mandela MarketPlace's Health and Wealth Net (MHWN) was developed to improve health-related quality of life and to increase access to economic opportunities by meeting community-identified food and wellness needs in Oakland.

Target Audience: MHWN focuses on the 23 census tracts identified as Vulnerable Populations in Alameda County; this includes 30,858 residents $<100 \%$ FPL.

Theory, Prior Research, Rationale: The MHWN Theory of Change views food insecurity and poor health indicators as attributable to poverty and social inequality. The model addresses this linkage and research on food deserts and health disparities by changing the food system to both prioritize ownership and asset-building and to increase access to healthy food for low income residents.

Description: The MHWN model is a healthy retail initiative that builds community-institution partnerships, strengthens local retail, and increases access to locallysourced produce. Through the incubation of an in-house produce distribution company and a cooperative grocery store, which source sustainably-grown produce from a network of local farmers, and the development of 16 new retail locations in targeted communities, MHWN is building a locally-owned network of healthy food-based businesses in Oakland.

Evaluation: As part of a Centers for Disease Control and Prevention REACH grant, a mixed-methods evaluation was conducted to determine change in amount of healthy foods purchased, estimate contribution of the model to the local economy, characterize the customers buying healthy foods and examine perceived benefits, challenges, impacts of participating in the MHWN.

Conclusions and Implications: Preliminary findings suggest that the model reaches the target audience, contributes substantively to the local economy, and successfully increases healthy food purchasing. Future evaluation should examine whether long-term objectives are successfully met.

Funding: Center for Disease Control and Prevention

\section{Examining the Need for a Healthy Corner Store Intervention}

Vanessa Berthoumieux, BS, University of Florida; Nicole Owens, $P h D$, University of Florida;

Karla Shelnutt, PhD, RD, kpagan@ufl.edu, University of Florida, 3028 McCarty Hall D, Gainesville, FL 32611

Objective: To determine the need for a healthy corner store intervention in food deserts.

Study Design, Setting, Participants: Geographic information systems (GIS) mapping, windshield surveys, semi-structured interviews with corner store managers $(n=2)$, food and marketing and walkability audits of recruited stores $(n=2)$, and customer intercept surveys $(n=60)$ were conducted in food deserts $(n=3)$ in one Florida county.

Outcome Measures and Analysis: GIS mapping: SNAP household density and proximity to corner stores; windshield surveys: store proximity to housing; food, marketing, and walkability audits: "healthfulness" ratings; manager interviews: perceived barriers to program success; customer intercept surveys: store accessibility, food purchasing/consumption habits, and food assistance program participation. Store audit data, store manager interviews, and customer intercept surveys were analyzed using Excel, Microsoft Word, and SPSS, respectively.

Results: Corner stores scored low on food, marketing, and walkability audits. Managers were uncertain whether customers would spend SNAP benefits on produce or other healthy items. Half of interviewd customers do not own a car, 62\% shop daily at the corner stores, and 58\% use SNAP benefits at the corner store. About half of customers eat three or more servings of fruits and vegetables per day. Participants would purchase fruits from the stores if available. Taste, healthfulness, and convenience had the largest impact on decision to purchase fresh fruits and vegetables.

Conclusions and Implications: SNAP-eligible residents living in food deserts purchase and consume food from the corner stores in their neighborhood and are interested in purchasing fruit if available. A healthy 
O27 (continued)

intervention would increase the availability of healthy foods in these food deserts.

Funding: University of Florida

\section{Influencing the Child Shopper with Coupons in Corner Stores: The CHOMPS Pilot Project}

Megan E. Lehnerd, MS, megan.lehnerd@tufts.edu, Tufts University, 150 Harrison Avenue, Boston, MA 02111; Anna R. McAlister, PhD, Michigan State University; Christina D. Economos, PhD, Tufts University; W. L. Adamowicz, PhD, FRSC, University of Alberta; Suzanne R. Howell, MPH, MS, Tufts University; Kaela Plank; Allison L. Gallop, MS, RD, CDE; Sean B. Cash, PhD

Objective: Evidence shows that children's independent food purchases in convenience stores are primarily purchases of energy-dense, nutrient-poor (EDNP) foods. Additional research is needed to understand how shifting children's snack food purchases could improve their diets.

Study Design, Setting, Participants, Intervention: This multi-disciplinary intervention utilizes economic, psychological, and nutritional insights to explore children's autonomous food purchase behaviors. Set in Somerville and Medford, MA, the intervention partnered with convenience stores near schools where kids shopped during out-of-school time. During our two-year, USDA-funded pilot, the Coupons for Healthier Options for Minors Purchasing Snacks (CHOMPS) study placed kids-only coupons in convenience stores with the goal of encouraging healthier snack purchases. Healthier snack coupons of varying discounts were paired with simple economic and health marketing messages.

Outcome Measures and Analysis: During before- and after-school times, data were recorded about the snack item, price, and other aspects of each purchase.

Results: Reported results include the observed purchase patterns of children, their responses to the coupon intervention in participating stores, and the nutritional composition of the observed purchases. Across more than 3,400 separate purchase events, we noted only modest coupon usage in the intervention phase. However, chip and candy purchases decreased, and the percentage of purchase events that included at least one targeted healthier food item more than doubled.

Conclusions and Implications: Results from this pilot indicate that kids-only coupons can assist with shifting snacking behavior outside of school settings. We anticipate that such an intervention would be even more impactful when combined with interventions focused on creating healthier corner stores - a strategy that is increasing in prominence.

Funding: USDA
029 Perspectives of Food Shoppers at Convenience Stores in Navajo Nation Food Deserts

Emily Piltch, MPH, BA, BS, emily.piltch@tufts.edu, Tufts University, 55 Los Gatos Circle, Sacramento, CA 95831; Tim Griffin, PhD, Tufts University; Robert F. Houser, PhD, Tufts University; Sonya Shin, MPH, MD, Community Outreach and Patient Empowerment (COPE); Carmen George, MS

Objective: Navajo face a double-burden of high rates of chronic disease and food insecurity, but little is known about the role small stores on the Navajo Nation play in providing healthy, affordable food. This study sought to understand barriers to and facilitators of purchasing produce at these stores.

Study Design, Setting, Participants: July to September 2016, a convenience sample of 72 shoppers participated in brief interviews outside eleven small stores in the New Mexico region of Navajo Nation.

Outcome Measures and Analysis: Shoppers discussed their shopping patterns, preferences and also food availability at the small store.

Results: Most participants (64\%) shopped primarily at bigbox stores outside their communities, yet about half shopped at the small store at least twice per week. The most common reasons for shopping at the small store were convenience to home/work and fuel purchase. The most commonly purchased items were snacks (42\%), staples (26\%), and soda (25\%). Almost half of participants worried in the last month about having enough food to feed their families and said that at least one person in the household participated in a federal assistance program. Shoppers would be more inclined to purchase produce if a greater variety of produce were available at affordable prices.

Conclusions and Implications: While small stores are the only retail options close to home for many Navajo, purchases are primarily snacks, rarely produce. The current and potential roles of small stores in food purchasing behaviors in rural communities will be discussed. This work provides basis for supporting innovative, community-based strategies for increasing access to healthy food close to home.

Funding: USDA

\section{Use of the Socioecological Model to Identify Factors of an Obesogenic Environment in Families with Young Children in Mexico}

Samantha Ramsay, PhD, LDN, RD, sramsay@uidaho.edu, University of Idaho, 875 Perimeter Drive, MS 3183, Moscow, ID 83844-3183; Jennie Davis, MS, University of Idaho; Lauren Keeney, MS; Samantha Worden, MS; Siew Guan Lee, RD, MS; Mackenzie Ferrante, MS, RD, Colorado State University; Jocelyn Macias, LN, Universidad Autónoma de Guadalajara; Delores Loaiza Lizarraga, PhD, $R D$

Objective: To identify factors related to an obesogenic environment in a small sample of families with young

Continued on page $S 13$ 